\title{
INVESTIGATIONS ON THIXOJOINING PROCESS OF STEEL COMPONENTS
}

\author{
M.N. Mohammed ${ }^{1}$, M.Z. Omar ${ }^{1 *}$, J. Syarif ${ }^{1}$, Z. Sajuri ${ }^{1}$, M.S. Salleh ${ }^{1}$ and \\ K.S. Alhawari ${ }^{1}$ \\ ${ }^{1}$ Department of Mechanical and Materials Engineering, \\ Faculty of Engineering and Built Environment, \\ Universiti Kebangsaan Malaysia, Malaysia \\ *Email: zaidi5886@gmail.com, Phone: +60192825906
}

\begin{abstract}
A high quality joint between tool steel and stainless steel alloys is hard to achieve with the conventional welding process. In this study, a new process of joining semi-solid AISI D2 tool steel and AISI 304 stainless steel using a partial remelting method is proposed. Metallographic analysis and Vickers hardness tests were performed. The results obtained from investigating the basic geometries demonstrated a good joining quality that differs from the conventional process of welding or mechanical joining with screws or nails. Metallographic analyses along the joint interface between semi-solid AISI D2 and 304 stainless steel showed a smooth transition from one to the other, with neither oxides nor microcracking being observed. Local mechanical properties of different regions show three different readings according to the results of the hardness tests. The current work successfully confirmed that the avoidance of dendritic microstructure of the semi-solid joined zone and high bonding quality components can be achieved. From the present study, it can be said that the thixojoining process can be utilized safely for dissimilar metal pairs.
\end{abstract}

Keywords: Thixojoining; direct partial remelting; non-equilibrium diffusion; JMatPro software.

\section{INTRODUCTION}

Semi-solid metal (SSM) processing is a comparatively new technology that involves the formation of metal alloys between the solidus and liquidus temperatures. Due to the emergence of thixotropic materials, many diverse techniques have been created for the purpose of utilizing the possibilities that arise from the characteristic benefits or advantages of this technology (Fan, 2002; Mohammed, Omar, Salleh, Alhawari, \& Kapranos, 2013; Salleh, Omar, Syarif, \& Mohammed, 2013). A key step for the successful operation of a semi-solid processing is the appropriate production of feedstock with a thixotropic property. In general, the partial remelting method is considered one of the most effective solid state processes, especially for high melting point metals, to produce non-dendritic microstructure when the metal is directly heated into the temperatures between solidus and liquidus (Mohammed, Omar, Syarif, et al., 2013; Omar, Alfan, Syarif, \& Atkinson, 2011). Metals in a thixotropic behavior have many features such as high softness, good flowability, superb workability and excellent joinability (Domínguez Almaraz, Guzmán Tapia, Tapia Silva, \& Cadenas Calderón, 2010; Kiuchi, Yanagimoto, \& Sugiyama, 2002). 
The thixojoining process is one of the forming operations in the thixoforming family. This technique has various advantages compared with conventional joining methods, including the possibility of producing functional components with multi materials, and minimizing the defects related to the conventional welding process. In addition, a semi-solid join can be used to join a low melting point metal with a high melting point metal (Kiuchi, Yanagimoto, \& Sugiyama, 1998; Ridha, Fonna, Huzni, Supardi, \& Ariffin, 2013; Yusof, Jamaludin, Abdullah, Hanafi, \& Zain, 2012). However, as reported in other studies, there are three possible methods to produce the thixojoining process: combining the forming and bonding as one step (Baadjou, Shimahara, \& Hirt, 2006), using a thixotropic metal as the filler for joining materials (Mendez, Rice, \& Brown, 2002), and joining two metals in a thixotropic property (Mohammed, Omar, Salleh, Zailani, \& Alhawari, 2012). The aims of the present study were to investigate the prospect of joining D2 tool steel to AISI 304 stainless steel and to characterize the interfacial microstructure and metallurgical joints which are accomplished in this novel process.

\section{EXPERIMENTAL MATERIAL AND PROCESS}

The material used in this work is AISI D2 cold work tool steel and AISI 304 stainless steel. The chemical compositions of the starting materials are given in Table 1.

Table 1. Chemical composition (wt\%) of the AISI D2 tool steel and 304 stainless steel by XRF technique.

\begin{tabular}{llllllllll}
\hline & $\mathrm{C}$ & $\mathrm{Si}$ & $\mathrm{Mn}$ & $\mathrm{Cr}$ & $\mathrm{Ni}$ & $\mathrm{Mo}$ & $\mathrm{V}$ & $\mathrm{P}$ & $\mathrm{S}$ \\
\hline AISI D2 & 1.46 & 0.258 & 0.239 & 11.2 & 0.197 & 0.769 & 0.711 & - & - \\
AISI 304 & 0.08 & 1 & 2 & 18 & 8 & - & - & 0.04 & 0.03 \\
\hline
\end{tabular}

The first process was concerned with cutting the AISI D2 (base material) into samples with a size of $22 \times 10 \times 5 \mathrm{~mm}$ and AISI 304 (insert) with a diameter of $\varnothing 6 \times 5$ $\mathrm{mm}$, as shown in Figure 1. Secondly, it was necessary to get rid of the rust and descale the surface by immersing the sample into a pickling solution with a concentration of $\left(40 \mathrm{ml} \mathrm{HCL}(37 \%)+60 \mathrm{ml} \mathrm{H}_{2} \mathrm{O}\right)$ for 5 minutes to get a fresh surface. Thirdly, the sample was washed in acetone, then rinsed with distilled water and dried in air.

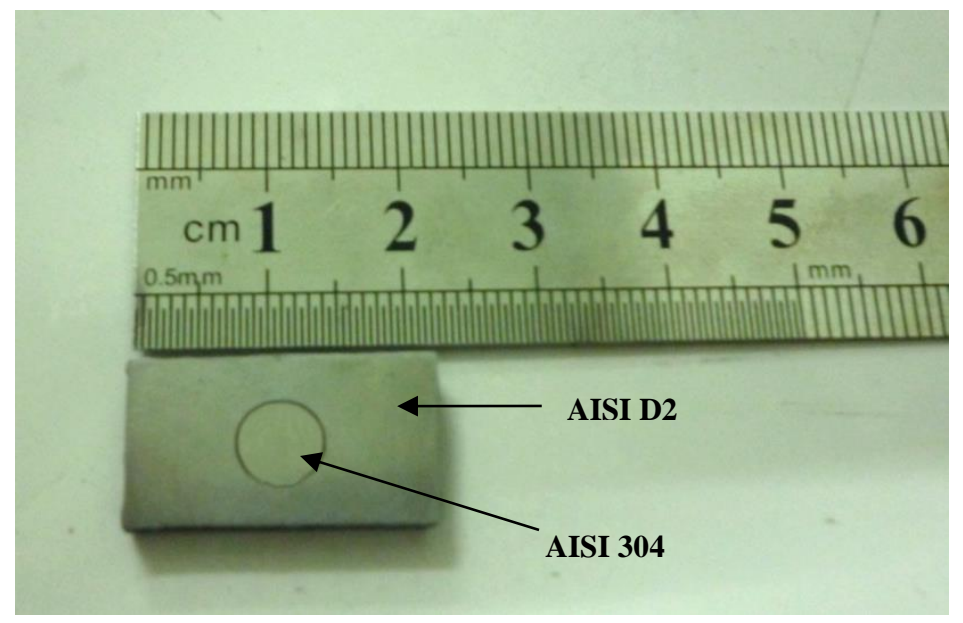

Figure 1. Combination of the two different materials. 
JMatPro (Java-based Material Properties) is a software solution that was developed to augment thermodynamic calculations by incorporating diverse theoretical material models and a properties database that enables a quantitative calculation of the requisite material's property to be made within a larger software structure (Salleh et al., 2013). Here, JMatPro software is used to estimate the solidus and liquidus temperatures as well as the liquid fraction profile within the semi-solid zone. The direct partial remelting experiment was performed using a vertical, high temperature carbolite furnace with a protective atmosphere of argon gas. Once the furnace reached the predefined temperature, the sample (base and insert) was lowered into the hottest point in the furnace, using a chromel wire into the targeted temperature $\left(1320^{\circ} \mathrm{C}\right)$, and held for 5 minutes, then cooled with an air-blow at room temperature, as shown in Figure 2. This ensures rapid heating of the coupon and it normally reaches the required temperature in about 5 minutes.

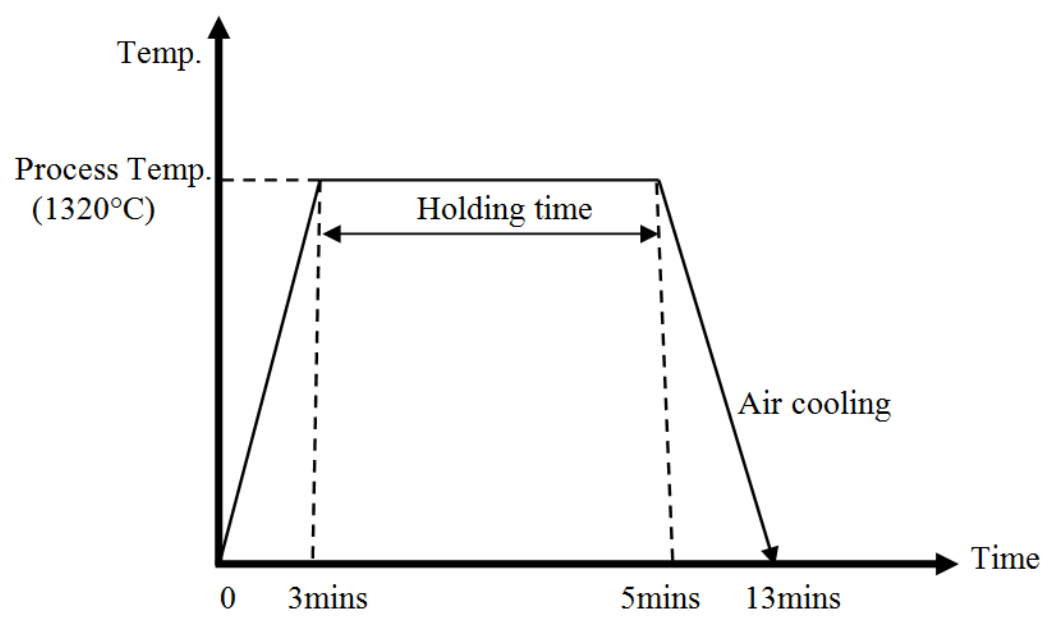

Figure 2. Temperature profile of sample inside the vertical tube furnace.

Samples from the direct partial remelting experiments were carefully ground and polished to obtain mirror surfaces. Microstructural characterization was carried out using a BX-51 Olympus optical microscope. All samples were etched using Villela reagent ( $1 \mathrm{~g}$ picric acid, $5 \mathrm{~mL}$ hydrochloric acid and $95 \mathrm{~mL}$ ethyl alcohol) to reveal their microstructures. Vickers hardness (HV) tests were accomplished on transverse sections. The hardness measurement profiles were performed through the sample's horizontal cross-section using a load of $2 \mathrm{~kg}$, applied for 15 seconds.

\section{RESULTS AND DISCUSSION}

\section{Microstructural Analysis}

The relationships between the liquid fraction, temperature and microstructure of AISI D2 are shown in Figure 3 by means of JMatPro Software. The liquid fraction profile (LFP) curve at $1320^{\circ} \mathrm{C}$ for AISI D2 is around 35\%, while the (LFP) for AISI 304 stainless steel is around $2.5 \%$. Thus there is an enormous difference between the liquid fraction percentages at the same temperature of the base metal and the insert. The optical microscopic photos taken from the thixo-welded joint are shown in Figure 4. According to the proposal by (Mohammed et al., 2012), the microstructures are created at the interface zone during direct partial remelting processes. Three main regions are 
apparent in Figure 4 that can be determined by the difference in color and grain size of the full penetration welded joint. It is clear that the eutectic liquid appears in the interface transition zone and a great quantity of the two metals' elements diffuse into each other to form new phases. As noted earlier, this is due to the huge difference between the liquid fraction percentages at the same temperature of the base metal and the insert.

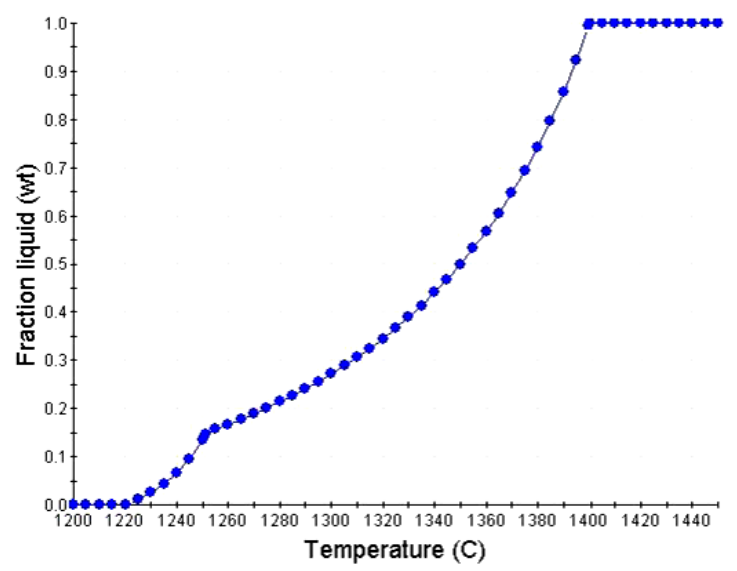

(a)

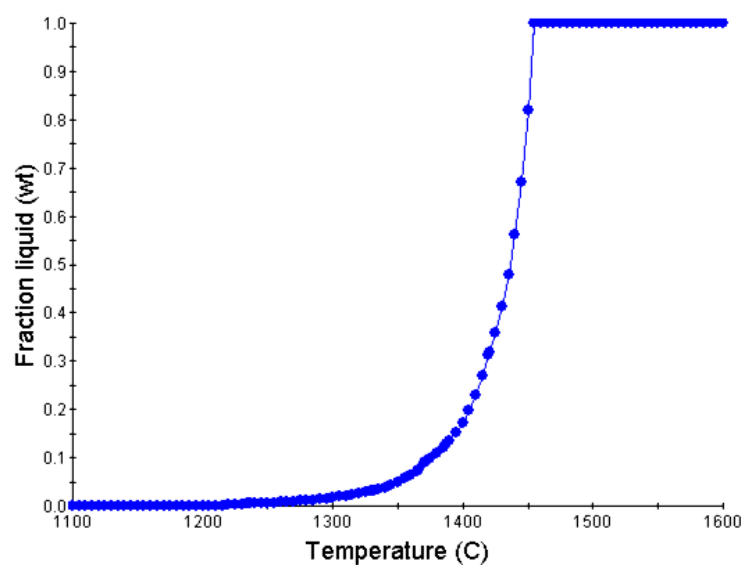

(b)

Figure 3. Liquid fraction profile with chemical composition of: (a) AISI D2 tool steel; and (b) AISI 304 stainless steel obtained from JMatPro simulation.
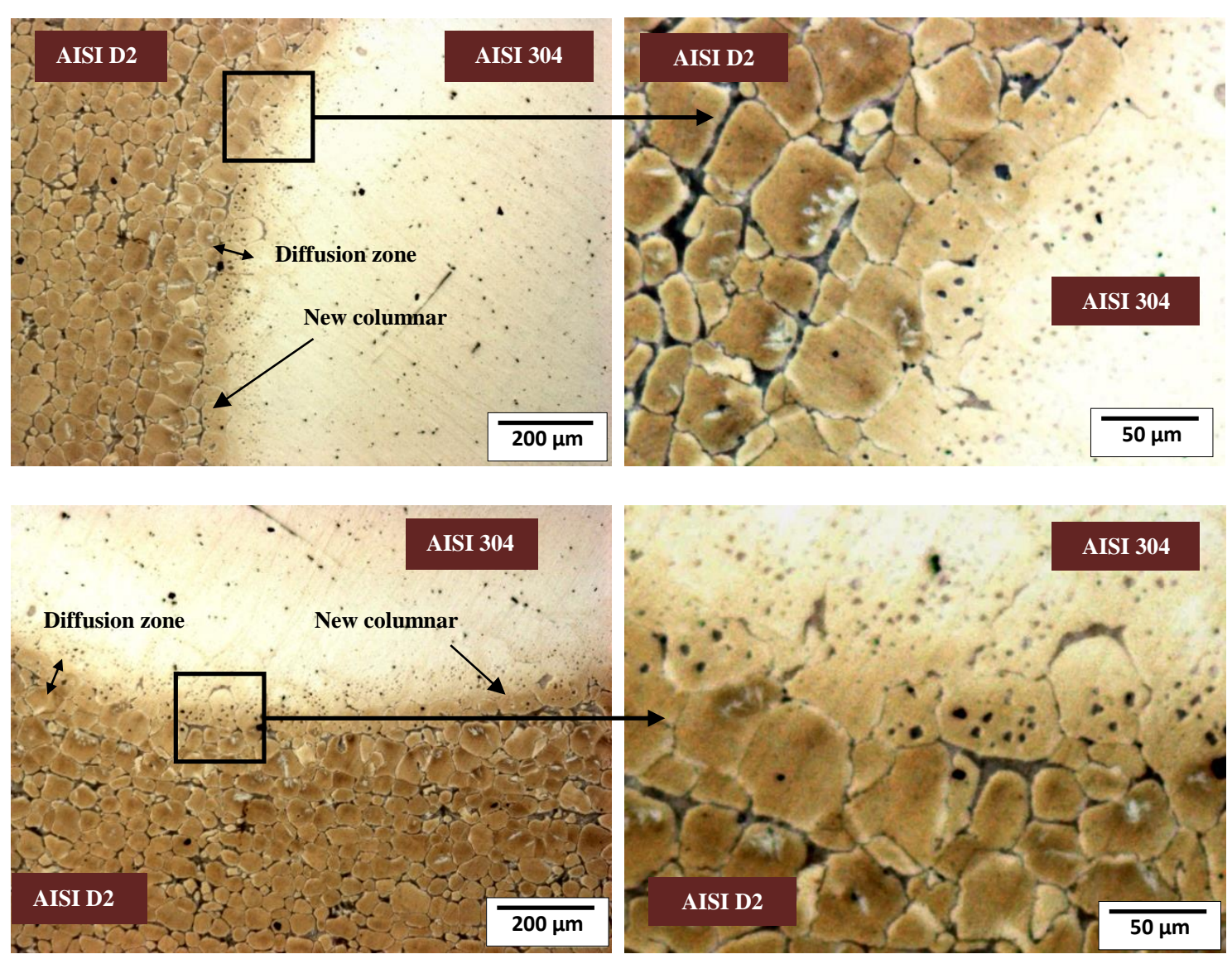

Figure 4. Microstructure of the joint interface of the AISI D2/AISI 304 pair. 
It can be seen clearly that there is a fine boundary between base blank and pin with a smooth transition of the diffusion zone from one to the other (Mohammed, Omar, Salleh, \& Alhawari, 2013). The transition occurs without any microcracking or porosity being detected, and the shape of the joint appears to be very smooth and not corrugated. Diffusion joining was also clearly seen from the grain boundary migration and the observation of different colours in single grains (Mohammed, Omar, Salleh, \& Alhawari, 2013). In addition, we can see the base metal grains changing as a result of the semi-solid processing to form new columnar crystals growing near the convergence zone (diffusion zone). During the construction of the interfacial structure, both metals, with their different reactions and diffusion ability between the solid and liquid forms of base metal, are connected with the insert along the bonding boundary, giving an indication of perfect joining at the interfaces of the products area.

\section{Vickers Hardness Testing}

The variation in the hardness values in the transverse cross-section of the joint is shown in Figure 5. It can be seen that the hardness values obtained from the points in the base metal are much higher than those in the insert. By contrast, in the diffusion zone the hardness value is higher than in the insert but lower than in the base metal. These differences are probably due to the diffusion of the elements from both metals at the interface zone of the joint. This hardness distribution data for the transverse crosssection of joints can be attributed to the fine transition structure between the base metal and the insert, as well as to the natural diffusion between these different metals.

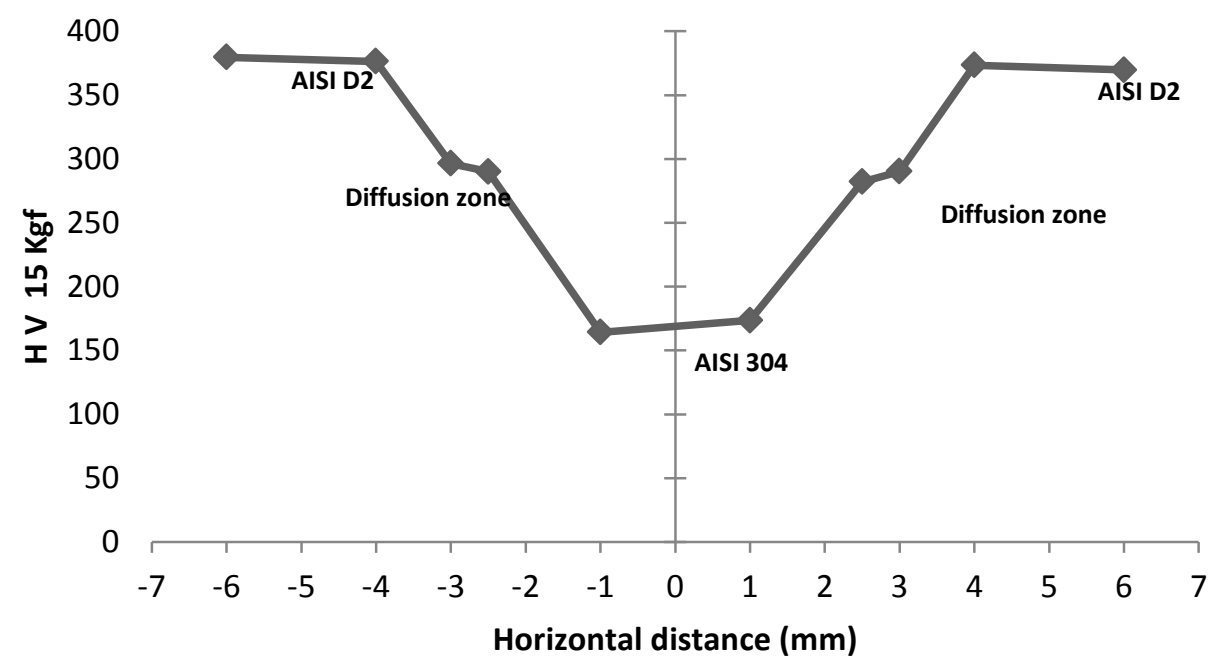

Figure 5. Vickers hardness profile.

\section{CONCLUSION}

Thixojoining has been successfully employed to weld two dissimilar steels by using a partial remelting method. Microstructural evaluation of the thixojoined joints shows three distinct zones, namely, the base metal AISI D2, the diffusion zone and the insert metal AISI 304. This technique was performed with fine interfacial diffusion along the bonding boundary between the base blank and insert. Furthermore, a smooth transition from one to the other occurred through the formation of new columnar crystals growing 
near the convergence zone. The transition occurs without any microcracking or porosity being detected. Hardness values obtained from the points in the diffusion zone were much higher than those in the AISI 304 stainless steel but lower than in the AISI D2 tool steel. These hardness variations of the three distinct zones can be explained by the diffusion of elements from both metals at the interface zone of the joint. From the present study it can be concluded that the thixojoining process can be used safely for different pairs of metals.

\section{ACKNOWLEDGEMENTS}

The authors would like to thank Universiti Kebangsaan Malaysia (UKM) and the Ministry of Higher Education (MOHE), Malaysia for the financial support under research grants: GUP-2012-040 and AP-2012-014.

\section{REFERENCES}

Baadjou, R., Shimahara, H., \& Hirt, G. (2006). Automated semi-solid forging of steel components by means of thixojoining. Solid State Phenomena, 116 - 117, 383386.

Domínguez Almaraz, G. M., Guzmán Tapia, M., Tapia Silva, E. E., \& Cadenas Calderón, E. (2010). Fatigue life prediction based on macroscopic plastic zone on fracture surface of aisi-sae 1018 steel. International Journal of Automotive and Mechanical Engineering, 1, 29-37.

Fan, Z. (2002). Semisolid metal processing. International Materials Reviews, 47(2), 4985.

Kiuchi, M., Yanagimoto, J., \& Sugiyama, S. (1998). Mashy-state joining, a new process for joining materials together. Paper presented at the 5th Int. Conf. Semi-Solid Processing of Alloys and Composites, Golden, Colorado, USA.

Kiuchi, M., Yanagimoto, J., \& Sugiyama, S. (2002). Application of mushy/semi-solid joining. Paper presented at the 7th Int. Conf. Semi-Solid Processing of Alloys and Composites, Tsukuba, Japan.

Mendez, P. F., Rice, C. S., \& Brown, S. B. (2002). Joining using semisolid metals. Welding Journal, 81(9), 181-187.

Mohammed, M. N., Omar, M. Z., Salleh, M. S., \& Alhawari, K. S. (2013). Study on thixojoining process using partial remelting method. Advances in Materials Science and Engineering, 2013, 1-8.

Mohammed, M. N., Omar, M. Z., Salleh, M. S., Alhawari, K. S., \& Kapranos, P. (2013). Semisolid metal processing techniques for nondendritic feedstock production. The Scientific World Journal, 2013, 1-16.

Mohammed, M. N., Omar, M. Z., Salleh, M. S., Zailani, M. A., \& Alhawari, K. S. (2012). Joining two metals via partial remelting method. Journal of Asian Scientific Research, 2(11), 724-730.

Mohammed, M. N., Omar, M. Z., Syarif, J., Sajuri, Z., Salleh, M. S., \& Alhawari, K. S. (2013). Microstructural evolution during dprm process of semisolid ledeburitic d2 tool steel. The Scientific World Journal, 2013, 1-7.

Omar, M. Z., Alfan, A., Syarif, J., \& Atkinson, H. V. (2011). Microstructural investigations of $\mathrm{xw}-42$ and $\mathrm{m} 2$ tool steels in semi-solid zones via direct partial remelting route. Journal of Materials Science, 46(24), 7696-7705. 
Ridha, M., Fonna, S., Huzni, S., Supardi, J., \& Ariffin, A. K. (2013). Atmospheric corrosion of structural steels exposed into the 2004 tsunami affected areas in aceh. International Journal of Automotive and Mechanical Engineering, 7, 1015-1023.

Salleh, M. S., Omar, M. Z., Syarif, J., \& Mohammed, M. N. (2013). Thermodynamic calculations of aluminium alloys for semi-solid processing. Paper presented at the 7th International Conference on Mathematics, Simulation, Modelling, Cambridge, MA, United States.

Yusof, M. F. M., Jamaludin, N., Abdullah, S., Hanafi, Z. H., \& Zain, M. S. M. (2012). Monitoring and assessment of acoustic emission signatures during fatigue mechanism of api5lx70 gas pipeline steel. Journal of Mechanical Engineering and Sciences, 2, 237-250. 